\title{
A Hidden Variable Model of Unstable Quantum States
}

\section{Bei G*}

Secondary School Teacher, IISS Charles Darwin, Italy

*Corresponding author: Gianpaolo Bei, Secondary School Teacher, IISS Charles Darwin, Via Tuscolana 388, Rome, Italy, Tel: +39 328 6779231; Email: capo.gian@libero.it

\section{Research Article}

Volume 5 Issue 1

Received Date: April 26, 2021

Published Date: May 10, 2021

DOI: $10.23880 /$ psbj-16000171

\section{Abstract}

We discuss the old problem of atom stability and atomic spontaneous emission, which was studied from Einstein introducing the idea of photon which was exploited by Bohr for its subsequent atomic model and its postulate of atomic quantum jumps. We expose then briefly the first historical model of unstable particles elaborated from Gamow which was based on the hypothesis of a constant lifetime describing the decaying process of unstable particles; we discuss then the relevance of this topic for our critique to the existence of hypothetical individual stationary atomic states. We propose a dynamic deterministic alternative approach to quantum unstable states and we suggest a possible alternative justification of Bohr formula of quantized energy spectra of the hydrogen atom. We assume that each atom is an open state that it is continuously radiating and interacting with the other accelerated atoms and that the stationary atomic spectra are an emergent effect caused by the equilibrium with the real electromagnetic pilot wave. We suggest consequently that the hydrogen gas is, with respect to each atom, an effective space time dependent polarized medium with a hidden variable light speed. We generalize the classical formula of spontaneous emission of Einstein and that one of Gamow substituting the standard light speed with a space dependent one. Finally we attempt to extend this approach to every atomic states and we propose a dynamical model of stationary quantum states that could allow to explain atomic stability and deduce dynamically the Bohr formula.

Keywords: Spontaneous Emission Rate; Unstable Quantum State; Hidden Variables; Variable Light Speed

\section{Introduction}

Soon after the formulation of the Bohr atomic model, based on the Einstein idea of the photon in 1905 (which he deduced, studying the photoelectric effect from statistical arguments), it emerged a great discussion on the problem of the radiation of the accelerated electron. In particular, it lacked a dynamic justification of the Bohr postulate of atomic quantum jumps and a clear deduction that formally could explain why the electron did not collapse in the nucleus. In 1917 Einstein [1] formulated a semi-classical model of atomic transitions based on the introduction of the empirical concept of a spontaneous emission rate which was connected to the induced emission rate and consequently to the ability of the atom to interact with the contextual electromagnetic fields of the other accelerating atoms

$$
A_{12}=\frac{8 \pi h v_{12}^{3}}{c^{3}} B_{12}(1)
$$

where A and B are the Einstein coefficients of spontaneous and induced emission and $v_{12}=\frac{E_{1}-E_{2}}{h}$ is the frequency of quantum jumps of the atomic stationary energy states.

This theory is a phenomenological one and its deduction was based on the Planck distribution law of energy density.

$$
\rho\left(v_{12}\right)=\frac{8 \pi h v_{12}^{3}}{c^{3}} \frac{1}{e^{h v_{12} / k T_{-1}}}
$$




\section{Physical Science \& Biophysics Journal}

It is evident that both formulas are statistical ones and that they assume that light speed is the usual constant phase velocity. At that time it was not clear that the assumption of validity of the relativity postulate of light speed constancy (experimentally verified only for classical electromagnetic fields in vacuum) was not rigorously justified for the hidden atomic transitions. In fact the Einstein energy formula of the indivisible photon was in contradiction with the Maxwell theory of electromagnetic fields. Over more, even the assumption of thermodynamic equilibrium used by Planck and Einstein was inadequate, since the accelerated excited atoms (and their associated electromagnetic fields) are presumed to be occupying energy levels in accordance with the Boltzmann equilibrium law.

$$
\frac{N_{1}}{N_{2}}=e^{-h v_{12} / k T}
$$

Therefore we think that the old idea of stationary individual atomic states is fallacious since it is based on the unjustified extension of the Maxwell theory and thermodynamic equilibrium to an ensemble of accelerated atoms. Subsequently, in 1928, just two years later after the publication by Schrodinger of its famous equation [2] the physicist G. Gamow introduced the first tentative model of quantum decay phenomena and unstable states [3] which applied then with success to his pioneering model of nuclear reactions. This was a non-relativistic model of unstable states, called Gamow kets, which was then applied in particles physics to the decay life of scattered particles and resonances observed in accelerators. We think that since then it has been underestimated the role of the electromagnetic fields and forces on the emergence of quantum mechanics and its statistics. In particular we believe that the energy spectra of single atoms are space-time dependent and they emit continuously electromagnetic radiation with variable light speed. The stationary atomic spectra are, we suggest, the result of a statistical ensemble averages. We propose in the next section a simple model based on a generalization of the Einstein formula in a medium with variable light speed and the consequent space dependent generalization of the standard Gamow energy band width. Then we impose a condition which we think could give a dynamical interpretation of the statistical energy atomic states. In the final section we briefly discuss the connection of our proposal with the open problem of the formulation of the Schrodinger Equation in an accelerated frame and the emerging role of the entanglement for a theory of unstable quantum particles in accelerated frame [4-6].

\section{Model}

Our proposal starts from the problem of atomic spontaneous emission by introducing a hidden light speed variable in the generalized Einstein spontaneous emission coefficients

$$
A_{i j}=A_{i j}(c(x, t))
$$

which of course implies a space-time dependence of atomic energy state. Therefore we can extend the Gamow energy band with formula in the following way

$$
E+i \Delta E(c(x, t))
$$

We impose then that the atomic states are stable if it is satisfied the following formula

$$
\nabla \Delta E=0
$$

This condition will determine, we presume, a set of discretized volumes, on which we can define a space-time average which can reproduce, once we find the hidden light speed variable, the Bohr formula of Hydrogen spectra.

$$
E_{k}(t)=\int_{0}^{v_{k}} \mu_{\varepsilon} d^{3} x(7)
$$

The model could be self-consistent once we found a discretized family of domains on whose borders is satisfied the formula (6), with is the energy density of the atomic electromagnetic fields defined by the following formula.

$$
\mu_{\varepsilon}=\frac{\varepsilon \vec{E}^{2}}{8 \pi}(8)
$$

We remark that the hidden electromagnetic field of the previous formula is a solution of the classical wave equation with a variable light speed and that the effective dielectric constant is dependent too on it. Then we assume that the variable light speed is coupled to the electric potential by the following formula (in analogy with the Einstein formula for gravitation light bending),

$$
n=1+\frac{2 \alpha \varphi}{c^{2}}
$$

where $n$ is the refraction's index of the atomic gas, $\alpha$ is an unknown phenomenological constant and is the electric potential solution of the classical wave equation. Finally we suggest that the hidden electric field could be equivalently defined by the following statistical average.

$$
\vec{E}=\int_{0}^{c} \nabla \phi\left(c^{\prime}\right) \rho\left(c^{\prime}, t\right) d c^{\prime}
$$

with $c^{\prime}$ a constant value of the light speed and $\rho$ an unknown probability density distribution.

Since we believe that our approach could be useful for reformulating the S.E.D. theory [7], we think that it could be tested the validity of the model, assuming that the density distribution is a Gaussian solution of the heat diffusion 
equation. With this choice we suggest that we could deduce the Bohr energy spectrum formula by the following temporal average.

$$
E_{k}^{B o h r}=\lim _{t \rightarrow+\infty} \frac{\int_{0}^{t} E_{k}\left(t^{\prime}\right) d t^{\prime}}{t}
$$

We think that our proposal could explain dynamically and deterministically the origin of quantum statistics and its connection with vacuum fluctuations and vacuum friction forces, as has been recently explored by some authors $[8,9]$. We want to remark that since the early researches of Planck and the subsequent alternative approach of Einstein, De Broglie, Schrodinger and Bohm against the Copenhagen interpretation of Quantum Mechanics, no model, as far as we know, has been proposed till now for explaining dynamically the origin of the Planck energy formula $=h v$. In fact we think that the photon hypothesis and the subsequent successful development of Q.E.D made superfluous the search of an interpretation of quantum states as an ensemble average of real oscillating electromagnetic fields. In particular we believe it is necessary to overcome the controversial concept of indivisible photon (which it is a monochromatic electromagnetic wave emitted and absorbed during atomic transitions) and that one of the wave particle dualism of Quantum Mechanics by introducing a real deterministic electromagnetic Poynting vector emitted by each atom.

We think it could be interesting to check the validity of our ideas by testing the Planck law in a free fall laboratory or in a rotating platform and measuring the supposed spacetime dependent spontaneous emission rate. We hope that our proposal of a dynamical formulation of atomic transitions will help to overcome the postulate of quantum wave function collapse which is the aim of recent active research on quantum decoherence called dynamical reduction models [10]. In particular we think that our approach could be useful for deterministic formulation of these models and sheds some light on the important open problem of the validity of the equivalence principle for quantum unstable particles [11] and, more generally, to unify quantum physics with gravity.

\section{Conclusion}

We propose in this paper a realistic simple model of unstable quantum states based on the hypothesis that the statistics of atomic transitions are caused by real atomic electromagnetic fields, whose fluctuations are caused by a hidden light speed variable. We generalize the Einstein spontaneous emission rate making it dependent on the variable light speed. We extend this realistic approach to every hydrogen atomic stationary state and we suggest a dynamical deduction of the Bohr atomic formula, interpreting stationary atomic states as emergent effect due to hidden macroscopic ensemble average. We propose then a dynamic electromagnetic condition at the base of atomic stability which could allow to reformulate deterministically, we hope, vacuum fluctuations. We suggest that a possible confirmation of our general approach would come in the future from the discovery of space- time environment dependent emission rates and from possible experimental violations of Planck law tested in accelerated laboratory frames.

\section{References}

1. Einstein A (1917) On the Quantum Theory of Radiation. Phys Z 18: 121.

2. Schrodinger E (1926) An Undulatory Theory of Mechanics of Atoms and Molecules. Phys Rew 28(6): 1049.

3. Gamov G (1928) Zur Quantentheorie des Atomkernes. Zeitschrift für Physik Z 51: 204-212.

4. Chakrabarty S (2020) Schroedinger Equation and the Associated Physics in Non-Inertial Frame. arxiv: 2002.12125.

5. Toros M, Restuccia S, Gibson MG, Cromb M, Ulbricht H, et al. (2020) Revealing and Concealing Entanglement with non-inertial motion. Phys Rew A 101(4): 043837.

6. Shi Y, Yang JC (2020) Entangled baryons: violation of inequalities based on local realism assuming dependence of decays on hidden variables. Eur Phys J C 80(2): 116.

7. de la Pena L, Cetto AM, Valdes-Hernandez A (2020) Connecting Two Stochastic Theories That Lead to Quantum Mechanics. Front Phys.

8. White H, Bailey P, Lawrence J, George J, Vera J (2020) A discussion on a dynamic vacuum model: Derivation of Helmholtz equation from Schrodinger equation. Physics Open 1: 100009.

9. Barnett SM, Sonnleitner M (2018) Vacuum friction. Journal of Modern Optics 65(1): 706-712.

10. Donadi S, Deckert DA, Bassi A (2014) On the spontaneous emission of electromagnetic radiation in the CSL Model. Ann Phys 340(1): 70-86.

11. Bonder Y, Fischbach E, Hernandez-Coronado H, Krause DE, Rohrbach Z, et al. (2013) Testing the Equivalence Principle with Unstable Particles. Phys Rew D 87(12): 125021. 